\section{$\underset{\substack{\text { hommes } \\ \text { \& migrations }}}{ }$}

\section{Hommes \& migrations}

Revue française de référence sur les dynamiques

migratoires

1289 | 2011

Les frontières du sport

\title{
Le Festival des Arts mélanésiens
}

\section{François Bensignor}

\section{(2) OpenEdition \\ Journals}

\section{Édition électronique}

URL : http://journals.openedition.org/hommesmigrations/818

DOI : 10.4000/hommesmigrations.818

ISSN : 2262-3353

\section{Éditeur}

Musée national de l'histoire de l'immigration

\section{Édition imprimée}

Date de publication : 1 janvier 2011

Pagination : 138-145

ISSN : 1142-852X

\section{Référence électronique}

François Bensignor, «Le Festival des Arts mélanésiens », Hommes \& migrations [En ligne], 1289 | 2011, mis en ligne le 29 mai 2013, consulté le 22 septembre 2020. URL : http://journals.openedition.org/ hommesmigrations/818; DOl : https://doi.org/10.4000/hommesmigrations.818

Ce document a été généré automatiquement le 22 septembre 2020.

Tous droits réservés 


\title{
Le Festival des Arts mélanésiens
}

\author{
François Bensignor
}

\section{NOTE DE L'ÉDITEUR}

Propos recueillis par François Bensignor

1 Le Festival des Arts mélanésiens est une initiative du Groupe du fer de lance mélanésien, créé au milieu des années quatre-vingt-dix et qui rassemble la PapouasieNouvelle-Guinée, les îles Salomon, la république de Fidji, Vanuatu et la NouvelleCalédonie via le Front de libération nationale kanak et socialiste (FLNKS). Cette dernière n'est pas membre du groupe, mais cherche à le devenir, c'est pourquoi elle a proposé d'accueillir la quatrième édition du festival en 2010. Les précédentes éditions ont eu lieu en 1998 aux îles Salomon, dans la capitale Honiara, à Port-Vila, capitale de la république de Vanuatu en 2002, puis à Suva, la capitale des îles Fidji en 2006.

François Bensignor: La thématique du festival s'articule autour de cette phrase: "Notre identité, elle est devant nous." Pourquoi ?

Emmanuel Kasarhérou: C'est une phrase de Jean-Marie Tjibaou qui invite à reformuler les limites identitaires et à les rendre plus poreuses qu'elles ne le sont habituellement dans un environnement insulaire. L'idée est de créer un brassage et de reconnaître la diversité culturelle qui existe aussi dans nos îles. Elle existe de manière très ancienne et quasi endémique, puisque cette partie du monde - la Mélanésie - contient un très petit nombre de populations - 8 millions de personnes mais qui parlent plus de 2000 langues parmi les 5000 répertoriées sur toute la planète. C'est une particularité humaine qui fait que les gens vivent dans une grande diversité culturelle depuis des milliers d'années. Cette diversité s'est enrichie du fait des phénomènes coloniaux et d'émigration contemporains. Or la façon ancienne de gérer la diversité culturelle offre sans doute des perspectives pour gérer la diversité d'un monde contemporain de plus en plus ouvert.

F. B. : La particularité de ce $4^{e}$ Festival des Arts mélanésiens est qu'il s'est démultiplié en quatre "pirogues". Ces entités festives itinérantes ont animé quatre territoires de la 
Nouvelle-Calédonie: le nord et le sud de la Grande-Terre, les îles Loyauté et la capitale Nouméa.

E. K. : On aurait été sous d'autres cieux, on les aurait appelées des caravanes... L'idée, c'est le voyage, la rencontre, faire en sorte de gérer des unités humaines qui soient suffisamment petites, mais également riches et denses en termes de programmation, afin que des relations humaines puissent s'établir. Les pirogues sont constituées de 120 à 250 personnes, ce qui permet de créer pendant une dizaine de jours une communauté humaine traversant le pays. C'est à la fois un voyage de présentation de ce que les participants ont amené, mais également une sorte de voyage initiatique au cœur d'un archipel mélanésien qui a ses propres singularités, ses particularités historiques. Notamment avec ses populations extrêmement variées, de souches européenne, indonésienne et vietnamienne, qui sont établies en Nouvelle-Calédonie depuis plus de cent ans, alors que d'autres îles sont mélanésiennes à plus de $90 \%$.

\section{F. B. : Comment le festival s'organise-t-il avec les autres pays?}

E. K. : Le principe est que chaque délégation nationale se constitue sur des critères les plus proches de ceux du pays organisateur. Chaque délégation nationale a la charge de constituer son équipe et de la transporter sur le lieu du festival, le pays organisateur se chargeant de tout à partir de là. Nous avions eu l'occasion d'inviter début 2010 les différentes personnes responsables de la constitution des délégations. Elles avaient pu constater la particularité de ce $4^{\mathrm{e}}$ Festival qui, au lieu de se dérouler sur un seul site, se déploie dans dix sites différents en l'espace de douze jours. Cela nécessite de la part des festivaliers d'accepter d'être sans arrêt sur la route. C'est ainsi qu'ont été choisies des équipes permettant des divisions par quatre. On a donc quatre unités autonomes [une par “pirogue"], qui représentent un élément particulier de la culture de chaque archipel, qu'il soit papou, salomonais, vanuatais ou fidjien. Ce parcours de petites unités sur la Nouvelle-Calédonie renforce aussi le sentiment de fierté culturelle des équipes qui sont réduites à vingt ou vingt-cinq personnes pour représenter tout un archipel dans sa diversité. Cette organisation a permis de favoriser les contacts avec un public calédonien finalement assez curieux de cette découverte.

F. B.: Quelles sont les principales tendances artistiques mises en valeur par les délégations?

E. K. : La danse et la musique jouent un rôle très important dans nos régions. D'ailleurs, on ne les distingue pas forcément. Parfois, des troupes de danse produisent essentiellement des chants en esquissant quelques petits pas. Il y a aussi des conteurs, des artisans qui font de la poterie, du tressage, etc. C'est une manière de montrer que dans certains champs de la culture, on a besoin de gens extrêmement spécialisés qui en font une profession, mais que dans d'autres, ce sont des gens qui exercent un métier: pêcheurs, planteurs, ou dans le secteur tertiaire d'une capitale. Chaque participant a toutefois un savoir-faire parce qu'il est porteur d'un patrimoine spécifique. Il s'agit donc d'une vision très élargie de ce que peut être l'art, dans ses éléments les plus élaborés comme dans les plus spontanés ou qui peuvent être partagés par un très grand nombre de gens.

\section{F. B. : Quelle est la proportion d'artistes professionnels?}

E. K. : Elle est relativement faible, dans la mesure où vivre de son art est quasiment impossible dans notre région, comme dans beaucoup d'autres régions du monde. On plante du manioc et l'on fait de la musique de temps en temps. Seuls les musiciens 
peuvent éventuellement vivre de leur art, parce que la musique dispose d'une petite économie. Mais cela ne concerne que très peu d'artistes.

F. B. : Quel est le dénominateur culturel à travers lequel les participants de ce festival se retrouvent?

E. K. : Le lien, c'est la diversité, même si ça peut paraître paradoxal. Il y a un tronc commun. Même quand les gens ont du mal à communiquer, ce qui est le cas dans un Pacifique essentiellement anglophone, les Calédoniens étant les seuls à parler français avec les Tahitiens et les Wallisiens. Le lien se fait essentiellement par les attitudes du corps, par tout ce qui est non verbal. La manière dont un individu se situe par rapport à un groupe révèle une grande proximité de vocabulaire de communication. Il y a une sorte d'imprégnation culturelle qui est propre à la Mélanésie. On constate que lorsqu'on fait venir des troupes constituées d'Aborigènes, le rapport au lieu et au groupe est très différent. Il existe également des proximités linguistiques, $d u$ fait que la plupart des langues sont soit papoues, soit austronésiennes. Elles ont un fonds lexical commun et des façons similaires d'organiser les idées, bien qu'il existe une diversité linguistique extraordinaire en Mélanésie. Dans certaines petites îles d'à peine $100 \mathrm{~km}$ de long sur $20 \mathrm{~km}$ de large au Vanuatu, 13 langues sont parlées par moins de 10000 personnes. En NouvelleCalédonie, on parle 28 langues alors que la population est inférieure à 100000 habitants. Ce qui est intéressant, c'est que ces langues continuent à se parler en même temps que d'autres. Le goût de la différenciation linguistique côtoie la volonté de se rassembler autour de langues plus universelles. Et le Festival des Arts mélanésiens est un moment où chaque groupe, fier de sa différence, marque son appartenance à une grande famille et jouit du cousinage qui l'unit aux autres peuples de la Mélanésie.

F. B.: Ce cousinage n'est-il pas particulièrement marquant dans le rapport que ces populations mélanésiennes entretiennent avec la nature et le cosmos?

E. K. : C'est sans doute l'aspect permanent. Il y a entre l'être vivant et celui qui a vécu une relation reposant sur une humanité qui ne serait pas complètement effacée de la planète et des lieux dans lesquels elle a vécu. La relation aux ancêtres et aux forces de la terre représente un trait consubstantiel aux diverses sociétés mélanésiennes. D'où ce très fort investissement dans tout ce qui est la terre, le lieu où l'on habite, où l'on pêche, où l'on prie. Les identités tournent autour de lieux de référence qui sont clairsemés sur l'ensemble de l'archipel comme des points qui révèlent un élément profond de l'individu, parce qu'en lui quelque chose porte cette histoire et cette relation entre le vécu qui n'est plus, celui qui se vit en ce moment et le lieu dans lequel ça se produit. Ici [au Centre culturel Tjibaou], on fait beaucoup de coutumes ${ }^{1}$ en rapport avec le lieu où l'on est, les gens qui sont là et ceux qui sont partis, etc. Or on retrouve des formes de coutumes tout à fait similaires dans toute la Mélanésie. Cet élément lié au lieu et au moment où l'on parle est d'ailleurs très océanien: on le retrouve jusqu'en Polynésie. Les langues sont organisées ainsi, beaucoup plus précises par rapport aux localisations que ne peuvent l'être les langues européennes. Lorsque l'on décrit quelque chose, il y a toujours des particules qui indiquent où est celui qui parle. En fonction de là où il est, il dit un certain nombre de choses sur son rapport aux autres. S'il est en bas ou en haut de la montagne, il n'emploie pas les mêmes particules. Ça dit aussi quelque chose sur son statut, sa position. Les langues traduisent cet enracinement, même s'il n'est que ponctuel. À ce moment-là, on fait 
une chose et le lendemain une autre, mais on cherche à chaque fois à trouver un enracinement dans le lieu où l'on est et le moment où l'on parle.

F. B. : L'histoire des tribus se raconte souvent par rapport aux pérégrinations des anciens...

E. K.: Oui, aux arrivées des pirogues, aux départs, etc. Sur la Grande-Terre, les populations sont plus enracinées que sur les îles Loyauté. Des montagnes - peut-être celles qui ont été vues par la vigie de la pirogue au moment où elle est arrivée - sont pensées comme des lieux d'origine. Chacun a son point d'atterrage et se situe dans le paysage par rapport à telle montagne, parce qu'il est originaire de la région qu'elle domine.

F. B.: L'influence des colons, notamment à travers la religion, se ressent-elle dans l'expression artistique des communautés mélanésiennes contemporaines?

E. K. : D'un point de vue musical, on sent une forte influence des cantiques religieux, des chants protestants ou de l'harmonium, dans la manière de tenir la note. Il y a chez les Kanaks un vrai goût pour le chant choral, la polyphonie vocale. Ce goût préexistait dans les traditions vocales mélanésiennes, mais sur les gammes non tempérées des chants " $a e-a e^{2 "}$ ". Quand les Mélanésiens ont découvert la gamme tempérée, ils ont laissé libre cours à leur imagination créative. Les chants ne sont d'ailleurs pas uniquement religieux. Chaque synode protestant est une occasion de créer de la musique, d'organiser des concours de chorales. Mais, par exemple, à l'occasion de l'ouverture du $4^{\mathrm{e}}$ Festival des Arts mélanésiens sur l'île de Maré [l'une des îles Loyauté], un chant spécifique a été créé par les membres de la tribu qui l'accueillait. C'est un art extrêmement vivant. L'imprégnation chrétienne est beaucoup plus forte dans les îles Loyauté que sur la Grande-Terre, parce qu'elles ont été christianisées 70 ans avant celle-ci. Les missionnaires sur la Grande-Terre venaient des îles Loyauté. L'influence religieuse est également très forte dans les autres archipels mélanésiens, comme Vanuatu. Souvent le motif musical est le même et les paroles changent d'une langue à l'autre. On retrouve, par exemple, l'adaptation de la musique de God Save the Queen à peu près dans toutes les langues, avec des paroles soit bibliques, soit qui n'ont rien à voir avec la religion : il peut s'agir de la dernière épidémie qui a décimé le village ou de la mort d'un chef.

F. B.: Le festival a été l'occasion de rencontres autour de thématiques propres à la Mélanésie, notamment sur les langues mélanésiennes au Centre culturel Tjibaou, mais aussi à Lifou lors d'un forum "Coutume et culture". Pouvez-vous nous en parler?

E. K. : Parmi les grandes particularités de la région mélanésienne, outre la profusion des langues dont certaines ne sont parlées que par six personnes, figure la relation à la coutume. Cette vision du monde articule les individus sur des tracés, des lignées, des relations à des groupes. On appelle "coutume" les modalités de communication entre les individus ou les groupes. Il s'agit de l'établissement de prises de parole : qui parle, qui ne peut pas parler... Le protocole varie selon les régions. Afin de préparer ce festival, il fallait connaître le protocole de Maré, de Lifou, d'Ouvéa, qui diffère dans le Nord et dans le Sud. Ces différentes façons de faire représentent des portes, qui sont manifestées par les modalités de prises de parole. Par où doit entrer la parole. C'est comme un chemin jalonné de passages. Il faut trouver la bonne passe dans la barrière de corail qui ceinture l'île. Il y a une passe pour les chefs, une pour les esprits, une pour les vivants qui ne sont pas chefs, etc. L'important est d'emprunter la bonne! 


\section{F. B. : Comment se discute la question de la coutume dans ce type de réunion?}

E. K. : Il ne s'agit surtout pas de niveler les différences pour parvenir à une sorte de texte commun. Chaque lieu et chaque histoire déterminent le protocole dans un lieu précis et avec des individus précis. On recherche plutôt la prise en compte de la parole coutumière, dans les prises de décisions nationales ou provinciales. Les représentants des îles Salomon ont évoqué, par exemple, le cas d'un lieu où il est parfaitementinconvenant de poser un relais téléphonique. L'État est en train de mettre en place un système de réseau mobile, mais les coutumiers refusent de voir installer des antennes dans certains lieux. Celles-ci gênent leur façon de concevoir dans leurs terroirs l'équilibre de ce qui est vivant, mais aussi de ce qu'on ne voit pas. Si le relais est installé malgré tout, il est rapidement détérioré. Par conséquent, les populations ne peuvent pas bénéficier du téléphone mobile. Il s'agit donc de voir dans quelle mesure une conciliation est possible. Au-delà du respect des croyances des individus, ce type de démarche permet aussi de préserver l'environnement : un facteur essentiel pour ces îles. Le colloque sur la coutume permet d'aborder ce genre de question. Des nations ont été implantées en Mélanésie depuis une trentaine d'années. Ces systèmes d'organisation peuvent être très efficaces, pourvu qu'ils entretiennent une relation intelligente avec ce qui préexiste.

F. B. : Le Centre culturel Tjibaou, que vous dirigez, développe tout un programme en relation avec les autorités coutumières, notamment en termes de collecte et d'archivage de la mémoire...

E. K. : Oui. La Nouvelle-Calédonie dispose d'un patrimoine immatériel considérable. Les mémoires orales sont très riches. On remonte facilement 300 ans, 400 ans en arrière, le maximum, daté avec précision grâce à des données archéologiques, étant de 500 ans. Le risque est que, face à d'autres centres d'intérêt, cette mémoire ne s'exerce plus de la même manière. Les gens sont également amenés à exercer leur mémoire sur d'autres savoir-faire nécessaires aujourd'hui, comme les études supérieures. C'est pourquoi nous avons mis au point au Centre Tjibaou une collecte généralisée du patrimoine oral. Elle est réalisée avec les conseils coutumiers. Chacun d'eux décide de ce qu'il estime être du domaine de la protection et l'on travaille avec des anciens pour enregistrer des généalogies, des mythes, des danses, des chants, etc. Chaque collecte est adaptée à la manière dont les gens du lieu envisagent les éléments menacés. Ces archives audio sont, dans un premier temps, destinées aux locuteurs de ces langues. Chaque texte est retranscrit et travaillé avec la personne qui a fourni l'information et fait l'objet d'au moins trois lectures critiques avec des locuteurs de la langue avant d'être archivé. Cinq personnes travaillent à temps plein sur ces collectes avec les conseils coutumiers. L'initiative a débuté en 2002. Elle s'est renforcée en 2006 et, aujourd'hui même, à l'occasion du symposium sur les langues mélanésiennes, nous rendrons accessibles sur Internet les résultats de nos collectes.

Festival des Arts mélanésiens : http://melanesia2010.nc

Centre culturel Tjibaou : www.adck.nc 


\section{NOTES}

1. Marie-Claude Tjibaou, présidente de l'Agence de développement de la culture kanak (ADCK) Centre culturel Tjibaou, explique: "Chez nous, la coutume est une pratique plus profonde que les simples salutations, qu'elle contient également. En général, lorsque l'on procède à la coutume, c'est un moment de silence qui permet l'écoute. On s'annonce, on dit l'objet de notre venue. On se retrouve les uns en face des autres et l'on se parle, avant d'entamer d'autres discussions. C'est un moment important : c'est là que l'on ouvre!"'“On écoute ce que l'autre dit. On prête attention à la personne qui parle. Celui qui répond prend la parole parce qu'il a l'aptitude de parler. Ce qu'il va dire est quelque chose qu'il a pensé, qu'il exprime au nom du groupe et qui doit être entendu par l'autre groupe. Tout le reste ira vite, si l'on a bien fait la coutume. Mais on ne peut pas faire la coutume n'importe comment. On attendra que toutes les personnes qui doivent y participer soient réunies pour commencer. La coutume doit être vécue par l'ensemble du groupe et la parole prononcée est destinée à l'ensemble des gens réunis là."

2. Origine des chants ae-ae selon Raymond Ammann, Danse et musiques kanak, ADCK, 1997, pp. 129-130: "Quelques informateurs de la région paicî disent que ce mot vient des mots paicî jawé et èa, qui évoquent 'le sourire de l'eau' [...]. Toutes les histoires, venant de différentes régions, sur la provenance des chants ae-ae ont en commun que la musique de ces chants a son origine dans l'eau. Dans la région de Poindimié, nous avons reçu l'histoire du vieux Baptiste de Tiéti selon laquelle, dans un creek, l'eau coulant sur un rocher a fait éclater celui-ci, et les chants ae-ae en sont sortis. Dans la région de Pouébo, nous avons entendu qu'un vieil homme rentrant de la pêche allait se laver au creek quand il entendit des voix. Quand il se retourna, il vit un mwake (un être spirituel, un esprit) assis sur un rocher, qui faisait s'entrechoquer deux pierres et chantait. Le vieux apprit ce prototype des chants ae-ae. Le vieux Marcel de Tioumidou (Poindimié) dit que son père a appris un chant un jour où il était assis à côté d'un creek pour se reposer et fumer une cigarette, car il avait entendu un chant qui montait de l'eau. Ainsi le vieux Ataba de Moméa (Moindou) a montré l'endroit du creek, au coin d'un rocher qui fait tourbillonner l'eau, d'où le prototype de son chant ae-ae est sorti."

\section{RÉSUMÉS}

Ce $4^{\mathrm{e}}$ Festival s'est déroulé en Nouvelle-Calédonie du 12 au 24 septembre 2010. Les manifestations, qui ont eu lieu sur l'ensemble du territoire de la Grande-Terre et des îles Loyauté, ont rassemblé un millier de participants, chanteurs, danseurs, artisans, détenteurs de savoirfaire traditionnels venus de tous les pays de l'arc mélanésien: Papouasie-Nouvelle-Guinée, îles Salomon, Fidji, Vanuatu, ainsi que du pays hôte la Nouvelle-Calédonie. L'organisation de ces douze jours de festivités, où les traditions mélanésiennes ont pu déployer leurs fabuleuses richesses sur une dizaine de sites, a été confiée au Centre Culturel Tjibaou. À quelques jours de la clôture de cet événement exceptionnel qui a attiré plus de 30000 spectateurs, son directeur, Emmanuel Kasarhérou, éclaire ses lignes de force en dégageant le sens profond de la démarche à la fois culturelle et festive qui a conduit sa réalisation. 